Advance Publication

\title{
The Journal of Veterinary Medical Science
}

Accepted Date: 3 February 2022

J-STAGE Advance Published Date: 16 February 2022

(C)2022 The Japanese Society of Veterinary Science

Author manuscripts have been peer reviewed and accepted for publication but have not yet been edited. 
Biochemistry

Full Paper

\section{Chondroitin sulfate $\mathbf{E}$ downregulates $\mathbf{N}$-cadherin and suppresses myotube formation}

Fumi Satoh $^{1}$, Akihiro Sugiura ${ }^{1}$, Jiro Tashiro ${ }^{1}$, Yoshinao Z. Hosaka ${ }^{1}$, Katsuhiko Warita ${ }^{1, *}$

${ }^{1}$ Department of Veterinary Anatomy, Faculty of Agriculture, Tottori University, Tottori 6808553, Japan

*Corresponding author: waritak@tottori-u.ac.jp

Department of Veterinary Anatomy,

Faculty of Agriculture, Tottori University,

4-101 Koyama Minami

Tottori $680-8553$

Japan

RUNNING HEAD: CHONDROITIN SULFATE E AND MYOGENESIS 


\begin{abstract}
Myogenesis, the formation of muscle fibers, is affected by certain glycoproteins, including chondroitin sulfate (CS), which are involved in various cellular processes. We aimed to investigate the mechanism underlying CS-E-induced suppression of myotube formation using the myoblast cell line C2C12. Differentiated cells treated with $0.1 \mathrm{mg} / \mathrm{ml} \mathrm{CS-E} \mathrm{for} \mathrm{nine} \mathrm{days}$ showed multinucleated and rounded myotubes with myosin heavy chain positivity. No difference was found between the CS-E-treated group with rounded myotubes and CS (-) controls with elongated myotubes in the levels of phospho-cofilin, a protein involved in the dynamics of actin cytoskeleton. Interestingly, N-cadherin, which is involved in the gene expression of myoblast fusion factors (myomaker and myomixer), was significantly downregulated at both the mRNA and protein levels following CS-E treatment. These results suggest that $\mathrm{N}$-cadherin downregulation is one of the mechanisms underlying the CS-E-induced suppression of myotube formation.
\end{abstract}

Keywords: chondroitin sulfate E, myoblast, myotube, N-cadherin 


\section{Introduction}

Proteoglycans are glycoproteins formed by the covalent binding of sulfated glycosaminoglycans (GAG) to a core protein and are involved in the formation of the extracellular matrix (ECM) $[16,17]$. Chondroitin sulfate (CS), a type of GAG, is composed of a repeated disaccharide sequence of D-glucuronic acid (GlcA) and N-acetyl-D-galactosamine (GalNAc), and is classified as CS-A, -B, -C, -D, -E, -K, and -H, depending on the number of sulfate groups and sulfated sites [25]. Due to the complexity of its molecular structure, CS participates in multifaceted physiological phenomena. It is not only involved in the formation of the ECM, but is also involved in various cellular phenomena, such as cell adhesion, migration, differentiation, and proliferation [21-23]. Furthermore, CS is localized on the cell surface where it exerts multiple physiological functions. For example, it acts as a receptor, promotes or inhibits the binding of humoral factors to receptors by binding to humoral factors such as cytokines, and induces intracellular signal transduction by binding to receptors as a ligand [21].

The skeletal muscle plays an important role in vital activities, such as motor function, energy metabolism, and glucose metabolism [7, 14, 32]. Fusion of myoblasts, which are precursors of myocytes, to form myotubes is essential for formation of skeletal muscle, also called myogenesis. Various proteins and regulatory mechanisms are involved in skeletal muscle formation from myoblasts. Myogenin, a muscle-specific transcription factor belonging to the myogenic regulatory factor (MRF) family, plays an important role in the regulation of genes involved in muscle differentiation and promotes myoblast differentiation [12, 31]. N-cadherin is a $\mathrm{Ca}^{2+}$-dependent intercellular adhesion factor known to regulate the expression of genes involved in muscle differentiation [6]. Similar to myomaker and myomixer, which fuse lipid bilayers between adjacent myoblasts, $\mathrm{N}$-cadherin, a protein expressed on the cell membrane, and $\beta$-catenin, which binds to the intracellular domain of $\mathrm{N}$-cadherin, regulate the transcription of factors involved in myoblast fusion $[7,8,13,20]$. Therefore, N-cadherin-induced 
intercellular adhesion plays an important role in skeletal muscle formation [6]. In contrast, cofilin 2 , an actin depolymerization factor specific to the skeletal muscle, is involved in the polymerization and depolymerization of actin filaments [29]. It has been reported that actin filaments are involved not only in the maintenance of the cytoskeleton and intracellular signal transduction, but also in muscle differentiation processes, such as cellular adhesion, cellular fusion, and changes in cellular morphology [29].

We had previously reported that CS-E, a hypersulfated CS chain, has the strongest inhibitory effect on myotube formation among CS-A, -B, -C, -D, and -E subtypes [33]. In this study, we hypothesized that the effects of CS-E on cell adhesion, via N-cadherin, and cytoskeletal reorganization, via cofilin 2 , may be one of the mechanisms by which CS-E suppresses myogenesis. Here, we show that suppression of CS-E-induced myotube formation is associated with a decrease in the expression level of factors related to cellular membrane fusion, especially $\mathrm{N}$-cadherin, rather than the expression levels of molecules involved in the polymerization and depolymerization of actin filaments.

\section{Materials and methods}

\section{Cell culture}

The mouse-derived myoblast cell line C2C12 (passage < 5; RIKEN BioResource Center, Tsukuba, Japan), which is capable of reproducing the differentiation process of the skeletal muscle, was used in this study. Cells were seeded at a density of $2 \times 10^{5}$ cells $/ \mathrm{ml}$ in 12 -well plates and incubated with Dulbecco's modified Eagle's medium (DMEM; Gibco, Thermo Fisher Scientific, Waltham, MA, USA) supplemented with $10 \%$ fetal bovine serum (FBS; Biosera, Ringmer, UK), 100 units/ml penicillin, and $100 \mu \mathrm{g} / \mathrm{ml}$ streptomycin (Fujifilm Wako Pure Chemical, Osaka, Japan) at $37{ }^{\circ} \mathrm{C}$ and $5 \% \mathrm{CO}_{2}$ until an $80 \%$ confluence was reached. 
The differentiation medium was DMEM containing 2\% horse serum (HS; Sigma-Aldrich, St. Louis, MO, USA), 100 units/ml penicillin, and $100 \mu \mathrm{g} / \mathrm{ml}$ streptomycin (Fujifilm Wako Pure Chemical). Differentiation induction was performed for nine days, starting from day 0 , the day when the medium was changed from DMEM with $10 \%$ FBS to DMEM with $2 \%$ HS. The differentiation medium was changed every three days to maintain optimal culture conditions. Each experimental data was obtained from at least three independent experiments.

\section{Analysis of the dose-dependent effects of CS-E}

Different concentrations of CS-E $(0.05,0.1,0.2$, and $0.5 \mathrm{mg} / \mathrm{ml})$ (PG Research, Tokyo, Japan) were added to the differentiation medium. The same volume of phosphate-buffered saline (PBS; Thermo Fisher Scientific), the vehicle for CS-E, was added to the control group. Following nine days of differentiation induction with each concentration of CS-E, the degree of muscle differentiation was evaluated immunocytochemically. The myosin heavy chain (MyHC)-positive area was defined as the myotube formation area. MyHC-positive regions were quantitated using the Image J software (National Institutes of Health, Bethesda, MD, USA). The myotube formation area relative to the total area of arbitrary five fields of view was compared among the five groups. For immunocytochemical analysis and gene/protein expression analysis, the control $(0 \mathrm{mg} / \mathrm{ml}$ of CS-E) and $0.1 \mathrm{mg} / \mathrm{ml} \mathrm{CS}$-E-treated group were designated as the CS (-) and CS (+) groups, respectively. The non-differentiated cells cultured with basal medium were designated as the ND group.

\section{Immunocytochemistry}

After differentiation induction, cells grown on coverslips in a 24-well plate were fixed with 2\% paraformaldehyde (Nacalai Tesque, Kyoto, Japan) for $30 \mathrm{~min}$, washed with PBS, and permeabilized with $0.1 \%$ Triton-X-100 (Nacalai Tesque) for 15 min. After washing with PBS, 
the cells were blocked with $2 \%$ bovine serum albumin (BSA) for 15 min to eliminate nonspecific reactions. An anti-mouse MyHC monoclonal antibody (MAB4470; R\&D Systems, Minneapolis, MN, USA) diluted at a ratio of 1:50 (final concentration $10 \mu \mathrm{g} / \mathrm{ml}$ ) in PBS was used as the primary antibody. Cells were incubated with the primary antibody for $1 \mathrm{hr}$ at room temperature. After washing with PBS, the cells were incubated with the secondary $\mathrm{CF}^{\mathrm{TM}}-568$ conjugated goat anti-mouse IgG antibody (Biotium, Hayward, CA, USA) diluted in PBS at a ratio of 1:200 for 15 min. Acti-stain ${ }^{\mathrm{TM}} 488$ Phalloidin (Cytoskeleton, Denver, CO, USA) was added to the secondary antibody reaction solution for F-actin staining. After washing with PBS, nuclear staining was performed using $5 \mu \mathrm{g} / \mathrm{ml}$ Hoechst 33342 (Dojindo, Kumamoto, Japan) for 15 min, and the slides were then mounted with Fluoromount/Plus ${ }^{\mathrm{TM}}$ (Diagnostic BioSystems, Pleasanton, CA, USA). Cells were analyzed using a Fluoview FV10i laser scanning confocal microscope (Olympus, Tokyo, Japan).

\section{Gene expression analysis of factors involved in myotube formation}

After differentiation induction for nine days, the culture medium was removed and total RNA was extracted from the cells using ISOSPIN Cell \& Tissue RNA (Nippon Gene Co., Tokyo, Japan). One microgram of total RNA was reverse transcribed into cDNA using the ReverTra Ace ${ }^{\circledR}$ qPCR RT Master Mix with gDNA Remover (Toyobo, Osaka, Japan). The gene expression levels of Myog (myogenin), Cfl2 (cofilin 2, muscle), Limk1 (LIM-domain containing, protein kinase), Ssh1 (slingshot protein phosphatase 1), Mymk (myomaker, myoblast fusion factor), Mymx (myomixer, myoblast fusion factor), and Cdh2 (cadherin 2, also known as N-cadherin) were analyzed using the DNA Green Master (Roche, Basel, Switzerland) and the LightCycler ${ }^{\circledR}$ Nano instrument (Roche). Gapdh (Glyceraldehyde-3-phosphate dehydrogenase) was used as the internal control. The primer sequences used in real-time PCR reactions are listed in Table 1. 


\section{Western blotting analysis}

After differentiation induction, the cells were washed with ice-cold PBS, and $100 \mu 1$ of CelLytic $^{\mathrm{TM}}$ M Cell Lysis Reagent (Sigma-Aldrich) supplemented with proteinase inhibitor (Sigma-Aldrich) was added to each well and placed on ice for $15 \mathrm{~min}$. The cell lysate was then collected and homogenized using a $27 \mathrm{G}$ needle. After centrifugation for $15 \mathrm{~min}$ at $4{ }^{\circ} \mathrm{C}$, the supernatant was collected. The bicinchoninic acid (BCA) method (BCA Protein Assay Reducing Agent Compatible; Thermo Fisher Scientific) was used for protein quantification. Electrophoresis was carried out using a NuPAGE ${ }^{\mathrm{TM}} 4-12 \%$ Bis-Tris Gel (Thermo Fisher Scientific) with $5 \mu \mathrm{g}$ of sample protein per well and PowerPac ${ }^{\mathrm{TM}} \mathrm{HC}$ (Bio-Rad, Hercules, CA, USA) at $200 \mathrm{~V}$ constant, $220 \mathrm{~mA}$, for $35 \mathrm{~min}$. Protein transfer to the nitrocellulose membrane was performed using an iBlot ${ }^{\circledR}$ Gel Transfer Device (Thermo Fisher Scientific). After protein transfer, the membrane was soaked in 5\% non-fat dry milk (Morinaga Milk Industry Co., Tokyo, Japan) and blocked for $1 \mathrm{hr}$ at room temperature. An anti-N-cadherin mouse monoclonal antibody (1:1,000, 610920; BD Biosciences, San Diego, CA, USA) and an anti-phospho-cofilin (Ser3) rabbit monoclonal antibody that nonspecifically recognizes phosphorylation of cofilin 1 and cofilin 2 (1:1,000, 77G2; Cell Signaling Technology, Danvers, MA, USA) were used as primary antibodies. An anti-GAPDH rabbit monoclonal antibody $(1: 1,000,14 \mathrm{C} 10$; Cell Signaling Technology) was used to detect the internal control GAPDH. After $1 \mathrm{hr}$ incubation with each primary antibody at room temperature, the membrane was washed with Tris-buffered saline containing $0.1 \%$ Tween 20 (TBS-T). Anti-mouse IgG goat antibody labeled with peroxidase $(1: 1,000, \mathrm{R} \& D$ Systems $)$ or anti-rabbit IgG goat antibody labeled with peroxidase (1:10,000, Kirkegaard \& Perry Laboratories, Gaithersburg, MD, USA) were used as secondary antibodies. The membrane was incubated with the secondary antibodies for $1 \mathrm{hr}$ at room temperature. Next, the nitrocellulose membrane was washed again and incubated with Clarity Western ECL substrate chemiluminescent detection reagent (Bio-Rad) for 5 min. Protein 
signals were detected using a C-DiGit Blot Scanner (Li-Cor Biosciences, Lincoln, NE, USA). Finally, the protein signals were quantified using the ImageJ software.

\section{Statistical analysis}

Statistical analysis was performed using Excel Statistics 2016 for Windows (version 3.21; SSRI, Tokyo, Japan). The Student's $t$-test (if homoscedasticity cannot be rejected) and Welch's $t$-test (upon rejection of homoscedasticity) were used to determine the significance of the differences between the two groups. One-way analysis of variance (ANOVA) with Bonferroni post-hoc test was used for multiple group comparisons. A $P$ value of $<0.05$ was considered statistically significant. 


\section{Results}

CS-E inhibits myotube formation and decreases myogenin gene expression

Immunocytochemical staining of $\mathrm{C} 2 \mathrm{C} 12$ cells cultured for nine days in differentiation medium supplemented with $0.05-0.5 \mathrm{mg} / \mathrm{ml} \mathrm{CS-E}$ showed that myotube elongation was suppressed and that MyHC-immunopositive areas were decreased compared to the control group (Fig. 1A). Immunocytochemical staining also revealed a significant $(P<0.01)$ decrease in MyHC-immunopositive areas in all CS-E-treated groups compared to those of the control group (Fig. 1B).

The gene expression level of the muscle-specific transcription factor myogenin showed a significant $(P<0.01)$ increase both in the CS $(-)$ and $(+)$ groups compared to that in the ND group (Fig. 1C). In contrast, a significant $(P<0.05)$ decrease in myogenin gene expression was observed in the CS (+) group compared to that in the CS (-) group.

\section{CS-E induced formation of round shaped myotubes}

Immunocytochemical staining images of MyHC and F-actin in the ND, CS (-), and CS $(+)$ groups are shown in Fig. 2. In the ND group, MyHC-negative cells with broad cytoplasm were observed (upper panel), whereas in the CS (-) group, MyHC-immunopositive, multinucleated, and elongated myotubes with fused cells were observed (middle panel). Similar to the CS (-) group, MyHC-immunopositive areas were also observed in the CS (+) group; however, many multinucleated myotubes with a round shape and no elongation were observed (Fig. 2, lower panel). In addition, the round myotubes had unclear phalloidin-positive actin fibers.

CS-E does not affect the expression of factors involved in actin remodeling

Real-time reverse transcription (RT)-PCR analysis of cofilin 2, an actin depolymerization 
factor involved in myotube elongation, showed no significant difference in the expression levels between the CS (-) and CS (+) groups (Fig. 3A). Furthermore, western blotting analysis showed no significant difference in the protein levels of phospho-cofilin (p-cofilin) between the two groups (Figs. 3B and 3C). The gene expression levels of Limk1 and Ssh1, the factors involved in cofilin protein phosphorylation and dephosphorylation, respectively, were not significantly different between the two groups (Figs. 3D and 3E).

\section{Round myotubes have fewer nuclei than elongated myotubes}

We arbitrarily selected 25 myotubes with elongated ( $\geq 500 \mu \mathrm{m}$ in length) or rounded shapes from a culture dish of the CS (-) and CS (+) groups, and compared the number of nuclei they contained. The fusion index (FI), which reflects the myotube fusion level, was determined by averaging the number of nuclei inside the myotubes. We found that elongated myotubes had significantly higher FI $(17.8 \pm 5.21)$ than round-shaped myotubes $(2.28 \pm 1.54)(P<0.01)$ (Fig. 4).

CS-E treatment of $C 2 C 12$ cells decreased the $N$-cadherin gene and protein expression levels

After differentiation induction, the mRNA level of the cell adhesion molecule N-cadherin was significantly decreased in the CS $(+)$ group compared to that in the CS $(-)$ group $(P<0.01)$ (Fig. 5A). Similarly, the N-cadherin protein levels were also significantly decreased following CS-E treatment $(P<0.05)$ (Figs. 5B and 5C).

CS-E treatment decreased the gene expression level of cell fusion-related factors in differentiation-induced C2C12 cells

The gene expression levels of myomaker and myomixer, factors involved in myoblast membrane fusion, are shown in Fig. 6. Compared to the ND group, differentiation-induced cells 
in the CS (-) and CS (+) groups showed a significantly higher myomaker and myomixer gene expression; however, the expression levels of these genes in the CS (+) group were significantly reduced compared to those in the CS (-) group $(P<0.01, P<0.05$, respectively). 


\section{Discussion}

CS contains a repeated disaccharide (GlcA and GalNAc) sequence, and is classified into several subtypes based on the number of sulfate groups added by chondroitin sulfotransferase. Compared to monosulfated CSs, such as CS-A, -B, and -C, the highly sulfated CSs, such as CS$\mathrm{E}$ and $-\mathrm{H}$, are known to be highly involved in the regulation of cell differentiation by acting on proteins, such as growth factors, and the ECM $[9,10]$. In fact, previous studies have shown that CS-E has a stronger effect on various cell physiological processes, such as cell differentiation and cell division, during development than other monosulfated CSs [23, 26, 27, 33].

Our previous study revealed that CS-E suppresses myotube formation during the differentiation process of $\mathrm{C} 2 \mathrm{C} 12$ cells, but its molecular mechanism was unclear [33]. In this study, we found that CS-E treatment significantly decreased the gene expression level of myogenin, suggesting that CS-E affected the differentiation of $\mathrm{C} 2 \mathrm{C} 12$ cells (Fig. 1C). In contrast, many round-shaped myotubes showing MyHC-positivity were observed in the CS-Etreated group (Figs. 1A and 2). These results suggest that CS-E, in addition to suppressing C2C12 cell differentiation, inhibits myotube elongation. In the CS-E-treated cells, obscure phalloidin-positive actin fibers were observed in the round-shaped myotubes. Therefore, we hypothesized that inhibition of actin polymerization or depolymerization was involved in the suppression of myotube elongation by CS-E, and focused on the effect of CS-E on cofilin.

Cofilin is a known actin-modulating protein whose function is regulated by its phosphorylation and dephosphorylation [28]. p-Cofilin, in which the third serine residue of cofilin is phosphorylated, is inactive, cannot bind to F-actin, and its depolymerization is inhibited [2]. In contrast, dephosphorylation of p-cofilin recovers its actin depolymerization activity. The depolymerization of F-actin supplies G-actin, which induces further polymerization [15]. LIMK is known to be involved in the phosphorylation of cofilin, whereas $\mathrm{SSH}$ is known to be involved in cofilin dephosphorylation. Their action is regulated in response 
to various cellular responses [30]. In this study, CS-E treatment did not affect p-cofilin or Limk, Ssh, and Cfl2 expression levels (Fig. 3). Hence, the CS-E-induced inhibition of myotube elongation was not related to actin remodeling inhibition.

Next, we focused on cell adhesion and fusion as the cause of myotube formation suppression. Comparing the FI in elongated $(\geq 500 \mu \mathrm{m})$ and round-shaped myotubes, FI in the round-shaped myotubes was significantly lower than that in the elongated myotubes (Fig. 4), suggesting that cell adhesion, fusion, or both are involved in the CS-E-induced suppression of myotube elongation. $\mathrm{N}$-cadherin is an intercellular adhesion molecule that accumulates at intercellular adhesion sites and functions in cell growth, differentiation, and organization during embryogenesis [5]. In this study, the mRNA and protein expression levels of N-cadherin were significantly reduced in the presence of CS-E (Fig. 5). Thus, CS-E suppressed the expression of $\mathrm{N}$-cadherin, which decreased the adhesive ability of $\mathrm{C} 2 \mathrm{C} 12$ cells and contributed to the inhibition of myotube formation. Koike et al. [18] reported that CS-E binds to N-cadherin and regulates intracellular signals in osteoblasts. The same phenomena may have occurred in the $\mathrm{C} 2 \mathrm{C} 12$ cells. Myomaker and myomixer are muscle-specific membrane proteins known to regulate myoblast fusion during embryogenesis $[3,24]$. Deletion of these genes has also been reported to inhibit myoblast fusion [34]. In myoblast fusion, myomaker acts on the cell membrane to bring the two cells closer together and cause semi-fusion. In contrast, myomixer forms fusion pores and expands them to complete intercellular fusion [4, 7]. Hence, both myomaker and myomixer play critical roles in myoblast fusion. In this study, the gene expression levels of myomaker and myomixer were significantly reduced in the presence of CS-E (Fig. 6). Thus, the fusion ability of $\mathrm{C} 2 \mathrm{C} 12$ cells likely decreased due to the CS-E-induced decrease in the expression levels of both myomaker and myomixer, and as a result, myotube formation was suppressed. In fact, it has been reported that knockout of myomaker and myomixer suppressed the formation of MyHC-positive multinucleated myotubes, which is 
consistent with our results [34]. The gene expression of proteins involved in membrane fusion, including myomaker and myomixer, is regulated by myogenin, which is known to bind to the promoter region of these genes and enhance gene expression $[1,11,19]$. In addition, myogenin is a muscle-specific transcription factor regulated by $\mathrm{N}$-cadherin-mediated intracellular signaling, and $\mathrm{N}$-cadherin inhibitors have been found to decrease myogenin protein expression in $\mathrm{C} 2 \mathrm{C} 12$ cells [6].

Collectively, evidence supports that reduction of N-cadherin by CS-E or binding of CS-E to $\mathrm{N}$-cadherin alters intracellular signaling, resulting in a decrease in myogenin gene expression and subsequent repression of myomaker and myomixer transcription, consequently inhibiting myotube formation (Fig. 7). These findings provide the foundation for developing novel approaches to muscle injury treatment. In this study, the CS-E-induced decrease in N-cadherin expression has been suggested to contribute to the inhibition of myotube formation; however, the mechanism underlying the CS-E-induced downregulation of $\mathrm{N}$-cadherin expression is unclear, and its elucidation is a topic for future research. 


\section{Acknowledgments}

This research was in part supported by JSPS Grant-in-Aid for Scientific Research (B) Grant Number JP17H03934.

\section{Competing interest}

The authors declare no competing financial interest. 


\section{References}

1. Adhikari, A., Kim, W. and Davie, J. 2021. Myogenin is required for assembly of the transcription machinery on muscle genes during skeletal muscle differentiation. PLoS One 16: e0245618. doi:10.1371/journal.pone.0245618.

2. Agnew, B. J., Minamide, L. S. and Bamburg, J. R. 1995. Reactivation of phosphorylated actin depolymerizing factor and identification of the regulatory site. J. Biol. Chem. 270: $17582-17587$.

3. Bi, P., McAnally, J. R., Shelton, J. M., Sánchez-Ortiz, E., Bassel-Duby, R. and Olson, E. N. 2018. Fusogenic micropeptide Myomixer is essential for satellite cell fusion and muscle regeneration. Proc. Natl. Acad. Sci. U. S. A. 115: 3864-3869.

4. Bi, P., Ramirez-Martinez, A., Li, H., Cannavino, J., McAnally, J. R., Shelton, J. M., Sánchez-Ortiz, E., Bassel-Duby, R. and Olson, E. N. 2017. Control of muscle formation by the fusogenic micropeptide myomixer. Science 356: 323-327.

5. Causeret, M., Taulet, N., Comunale, F., Favard, C. and Gauthier-Rouvière, C. 2005. Ncadherin association with lipid rafts regulates its dynamic assembly at cell-cell junctions in C2C12 myoblasts. Mol. Biol. Cell 16: 2168-2180.

6. Charrasse, S., Meriane, M., Comunale, F., Blangy, A. and Gauthier-Rouvière, C. 2002. $\mathrm{N}$-cadherin-dependent cell-cell contact regulates Rho GTPases and $\beta$-catenin localization in mouse C2C12 myoblasts. J. Cell Biol. 158: 953-965.

7. Chen, B., You, W., Wang, Y. and Shan, T. 2020. The regulatory of Myomaker and Myomixer-Myomerger-Minion in muscle development and regeneration. Cell. Mol. Life Sci. 77: 1551-1569.

8. Cui, S., Li, L., Yu, R. T., Downes, M., Evans, R. M., Hulin, J. A., Makarenkova, H. P. and Meech, R. 2019. $\beta$-Catenin is essential for differentiation of primary myoblasts via cooperation with MyoD and $\alpha$-catenin. Development 146: $\operatorname{dev} 167080$. 
doi:10.1242/dev.167080.

9. Deepa, S. S., Umehara, Y., Higashiyama, S., Itoh, N. and Sugahara, K. 2002. Specific molecular interactions of oversulfated chondroitin sulfate $\mathrm{E}$ with various heparin-binding growth factors. J. Biol. Chem. 277: 43707-43716.

10. Furutani, Y., Manabe, R., Tsutsui, K., Yamada, T., Sugimoto, N., Fukuda, S., Kawai, J., Sugiura, N., Kimata, K., Hayashizaki, Y. and Sekiguchi, K. 2005. Identification and characterization of photomedins: novel olfactomedin-domain-containing proteins with chondroitin-E-binding activity. Biochem. J. 389: 675-684.

11. Ganassi, M., Badodi, S., Quiroga, H. P. O., Zammit, P. S., Hinits, Y. and Hughes, S. M. 2018. Myogenin promotes myocyte fusion to balance fibre number and size. Nat. Commun. 9: 4232. doi:10.1038/s41467-018-06583-6.

12. Ganassi, M., Badodi, S., Wanders, K., Zammit, P. S. and Hughes, S. M. 2020. Myogenin is an essential regulator of adult myofibre growth and muscle stem cell homeostasis. Elife 9: e60445. doi:10.7554/eLife.60445.

13. Goichberg, P., Shtutman, M., Ben-Ze 'ev, A. and Geiger, B. 2001. Recruitment of $\beta$ catenin to cadherin-mediated intercellular adhesions is involved in myogenic induction. J. Cell Sci. 114: 1309-1319.

14. Hindi, S. M., Tajrishi, M. M. and Kumar, A. 2013. Signaling mechanisms in mammalian myoblast fusion. Sci. Signal. 6: re2. doi:10.1126/scisignal.2003832.

15. Huang, T. Y., DerMardirossian, C. and Bokoch, G. M. 2006. Cofilin phosphatases and regulation of actin dynamics. Curr. Opin. Cell Biol. 18: 26-31.

16. Karamanos, N. K., Piperigkou, Z., Theocharis, A. D., Watanabe, H., Franchi, M., Baud, S., Brézillon, S., Götte, M., Passi, A., Vigetti, D., Ricard-Blum, S., Sanderson, R. D., Neill, T. and Iozzo, R. V. 2018. Proteoglycan chemical diversity drives multifunctional cell regulation and therapeutics. Chem. Rev. 118: 9152-9232. 
17. Kitagawa, H. 2014. Using sugar remodeling to study chondroitin sulfate function. Biol. Pharm. Bull. 37: 1705-1712.

18. Koike, T., Izumikawa, T., Tamura, J. and Kitagawa, H. 2012. Chondroitin sulfate-E finetunes osteoblast differentiation via ERK1/2, Smad3 and Smad1/5/8 signaling by binding to N-cadherin and cadherin-11. Biochem. Biophys. Res. Commun. 420: 523-529.

19. Lehka, L. and Rędowicz, M. J. 2020. Mechanisms regulating myoblast fusion: a multilevel interplay. Semin. Cell Dev. Biol. 104: 81-92.

20. Leikina, E., Gamage, D. G., Prasad, V., Goykhberg, J., Crowe, M., Diao, J., Kozlov, M., M., Chernomordik, L. V. and Millay, D., P. 2018. Myomaker and Myomerger work independently to control distinct steps of membrane remodeling during myoblast fusion. Dev. Cell 46: 767-780.

21. Mikami, T. and Kitagawa, H. 2013. Biosynthesis and function of chondroitin sulfate. Biochim. Biophys. Acta 1830: 4719-4733.

22. Mikami, T. and Kitagawa, H. 2017. Sulfated glycosaminoglycans: their distinct roles in stem cell biology. Glycoconj. J. 34: 725-735.

23. Mikami, T., Koyama, S., Yabuta, Y. and Kitagawa, H. 2012. Chondroitin sulfate is a crucial determinant for skeletal muscle development/regeneration and improvement of muscular dystrophies. J. Biol. Chem. 287: 38531-38542.

24. Millay, D. P., O’Rourke, J. R., Sutherland, L. B., Bezprozvannaya, S., Shelton, J. M., Bassel-Duby, R. and Olson, E. N. 2013. Myomaker: a membrane activator of myoblast fusion and muscle formation. Nature 499: 301-305.

25. Miyachi, K., Wakao, M. and Suda, Y. 2015. Syntheses of chondroitin sulfate tetrasaccharide structures containing 4,6-disulfate patterns and analysis of their interaction with glycosaminoglycan-binding protein. Bioorg. Med. Chem. Lett. 25: 15521555 
26. Miyazaki, T., Miyauchi, S., Anada, T., Tawada, A. and Suzuki, O. 2015. Chondroitin sulfate-E binds to both osteoactivin and integrin $\alpha \mathrm{V} \beta 3$ and inhibits osteoclast differentiation. J. Cell. Biochem. 116: 2247-2257.

27. Miyazaki, T., Miyauchi, S., Tawada, A., Anada, T., Matsuzaki, S. and Suzuki, O. 2008. Oversulfated chondroitin sulfate-E binds to BMP-4 and enhances osteoblast differentiation. J. Cell. Physiol. 217: 769-777.

28. Moriyama, K., Iida, K. and Yahara, I. 1996. Phosphorylation of Ser-3 of cofilin regulates its essential function on actin. Genes Cells 1: 73-86.

29. Nguyen, M. T., Min, K. H., Kim, D., Park, S. Y. and Lee, W. 2020. CFL2 is an essential mediator for myogenic differentiation in $\mathrm{C} 2 \mathrm{C} 12$ myoblasts. Biochem. Biophys. Res. Commun. 533: 710-716.

30. Ohashi, K. 2015. Roles of cofilin in development and its mechanisms of regulation. Dev. Growth Differ. 57: 275-290.

31. Sampath, S. C., Sampath, S. C. and Millay, D. P. 2018. Myoblast fusion confusion: the resolution begins. Skelet. Muscle 8: 3. doi:10.1186/s13395-017-0149-3.

32. Vaz, R., Martins, G. G., Thorsteinsdóttir, S. and Rodrigues, G. 2012. Fibronectin promotes migration, alignment and fusion in an in vitro myoblast cell model. Cell Tissue Res. 348: 569-578.

33. Warita, K., Oshima, N., Takeda-Okuda, N., Tamura, J. and Hosaka, Y. Z. 2016. Degree of suppression of mouse myoblast cell line $\mathrm{C}_{2} \mathrm{C}_{12}$ differentiation varies according to chondroitin sulfate subtype. Mar. Drugs 14: 193. doi:10.3390/md14100193.

34. Zhang, H., Wen, J., Bigot, A., Chen, J., Shang, R., Mouly, V. and Bi, P. 2020. Human myotube formation is determined by MyoD-Myomixer/Myomaker axis. Sci. Adv. 6: eabc4062. doi:10.1126/sciadv.abc4062 


\section{Figure legends}

\section{Figure 1. Effect of chondroitin sulfate E (CS-E) on myotube formation}

(A) Immunocytochemical staining images of myosin heavy chain (MyHC) in $\mathrm{C} 2 \mathrm{C} 12$ cells following differentiation induction for nine days. CS-E induced a decrease in the MyHCpositive area. In addition, myotube elongation was suppressed in the CS-E-treated groups compared to the control. MyHC (red), nucleus (blue). Scale bar $=200 \mu \mathrm{m}$. (B) Quantification of MyHC-positive regions using the ImageJ software. The MyHC-positive area relative to the total area (arbitrary five fields of view) was compared among the five groups. (C) Myogenin mRNA expression levels in the non-differentiated (ND), $0 \mathrm{mg} / \mathrm{ml} \mathrm{CS-E}$, and $0.1 \mathrm{mg} / \mathrm{ml} \mathrm{CS-E}$ groups. Glyceraldehyde-3-phosphate dehydrogenase (Gapdh) was used as the internal control. Mean $\pm \mathrm{SD}, \mathrm{n}=3-5$, Boferroni post-hoc test, $* P<0.05, * * P<0.01$

\section{Figure 2. Chondroitin sulfate E (CS-E)-induced morphological changes in the myotubes} Confocal imaging of the myosin heavy chain (MyHC)-positive area and F-actin in C2C12 cells following differentiation induction for nine days. In the CS (-) group (0 mg/ml CS-E), elongated and multinucleated myotubes showing MyHC-positivity were observed. Both the CS (+) $(0.1 \mathrm{mg} / \mathrm{ml} \mathrm{CS}-\mathrm{E})$ and CS (-) groups exhibited MyHC-positive areas; however, the myotubes in the CS (+) group had a round shape. Nuclei (blue), MyHC (red), and F-actin (green). Scale bar $=50 \mu \mathrm{m}$.

Figure 3. Comparison of the mRNA and protein expression levels of molecules involved in actin remodeling

(A) Gene expression level of cofilin 2, involved in actin remodeling and myotube elongation.

(B) Western blotting analysis of the protein expression levels of phospho (p)-cofilin and glyceraldehyde-3-phosphate dehydrogenase (GAPDH) in the $0 \mathrm{mg} / \mathrm{ml}$ chondroitin sulfate E 
(CS-E) $[\mathrm{CS}(-)]$ and $0.1 \mathrm{mg} / \mathrm{ml} \mathrm{CS-E} \mathrm{[CS} \mathrm{(+)]} \mathrm{groups.} \mathrm{Band} \mathrm{intensity} \mathrm{was} \mathrm{quantified} \mathrm{using} \mathrm{the}$ ImageJ software (C). Comparison of the gene expression levels of Limk1 (D) and Ssh1 (E) between the CS (-) and CS (+) groups. Mean $\pm \mathrm{SD}, \mathrm{n}=3$, Welch's $t$-test (A) and Student's $t$ test (C, D, E), n.s.; not significant.

\section{Figure 4. Myotube length and number of nuclei contained in myotubes}

The number of nuclei contained in the myosin heavy chain (MyHC)-positive area was defined as the Fusion index (FI). The FI was calculated using 25 myotubes with elongated shape $(\geq 500$ $\mu \mathrm{m})$ from $0 \mathrm{mg} / \mathrm{ml}$ chondroitin sulfate $\mathrm{E}(\mathrm{CS}-\mathrm{E})[\mathrm{CS}(-)]$ group and 25 myotubes with rounded shape from $0.1 \mathrm{mg} / \mathrm{ml} \mathrm{CS-E} \mathrm{[CS} \mathrm{(+)]} \mathrm{group.} \mathrm{The} \mathrm{boxes} \mathrm{represent} \mathrm{the} \mathrm{distribution} \mathrm{of} \mathrm{the} \mathrm{number}$ of nuclei between the first quartile (Q1) and third quartile (Q3). The horizontal line between Q1 and Q3 represents the median $(\mathrm{Q} 2)$. “×” indicates the average value of the FI in each group. Outliers are not displayed. $\mathrm{n}=25, * * P<0.01$, Welch's $t$-test.

\section{Figure 5. Expression level of the cell adhesion molecule $\mathbf{N}$-cadherin}

Comparison of the $\mathrm{N}$-cadherin gene (A) and protein (B) expression levels between the $0 \mathrm{mg} / \mathrm{ml}$ chondroitin sulfate E (CS-E) [CS (-)] and $0.1 \mathrm{mg} / \mathrm{ml} \mathrm{CS-E} \mathrm{[CS} \mathrm{(+)]} \mathrm{groups.} \mathrm{Glyceraldehyde-}$ 3-phosphate dehydrogenase (GAPDH) was used as the internal control. Protein bands were quantified using the ImageJ software, and the relative quantitative values were determined (C). Mean $\pm \mathrm{SD}, \mathrm{n}=3$, Student's $t$-test, $* P<0.05, * * P<0.01$

\section{Figure 6. Comparison of the gene expression level of factors involved in cellular membrane fusion}

Comparison of the gene expression levels of myomaker (A) and myomixer (B) between the non-differentiated (ND), $0 \mathrm{mg} / \mathrm{ml}$ chondroitin sulfate E (CS-E) [CS (-)], and $0.1 \mathrm{mg} / \mathrm{ml} \mathrm{CS-E}$ 
[CS (+)] groups. Glyceraldehyde-3-phosphate dehydrogenase (Gapdh) was used as the internal control. The ND group showed extremely low expression levels of both myomaker and myomixer. Mean $\pm \mathrm{SD}, \mathrm{n}=3$, Bonferroni post-hoc test, ${ }^{*} P<0.05,{ }^{* *} P<0.01$.

\section{Figure 7. Illustration of the experimental results}

(1) Reduction of N-cadherin by chondroitin sulfate E (CS-E) or binding of CS-E to N-cadherin alters intracellular signaling. (2) Decreased myogenin expression. (3) The gene expression levels of myomaker and myomixer were decreased due to the decrease in the levels of myogenin, which binds to the promoter region of myomaker and myomixer, enhancing their expression. As a result, cell membrane fusion was inhibited. 
Figure 1

A
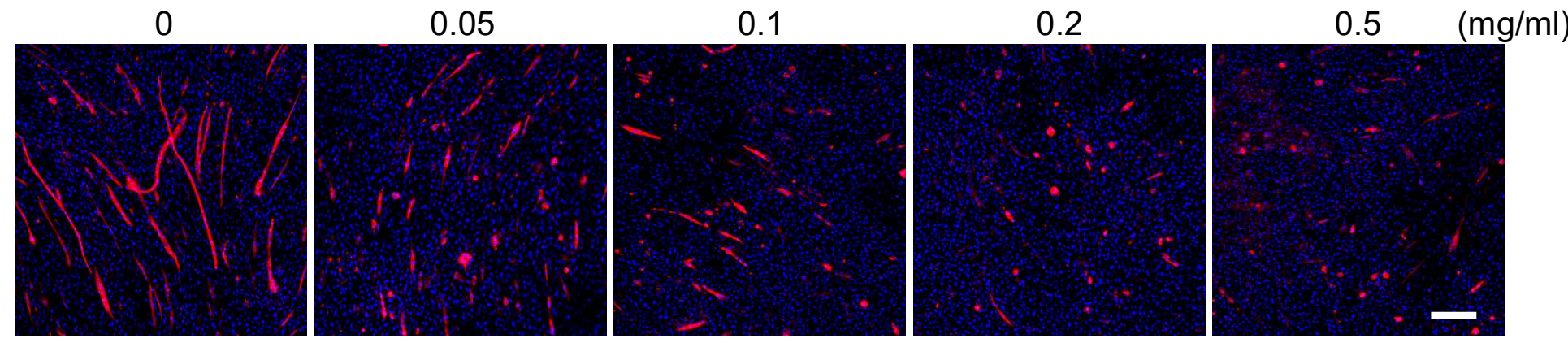

Red: MyHC

Blue: Hoechst

B

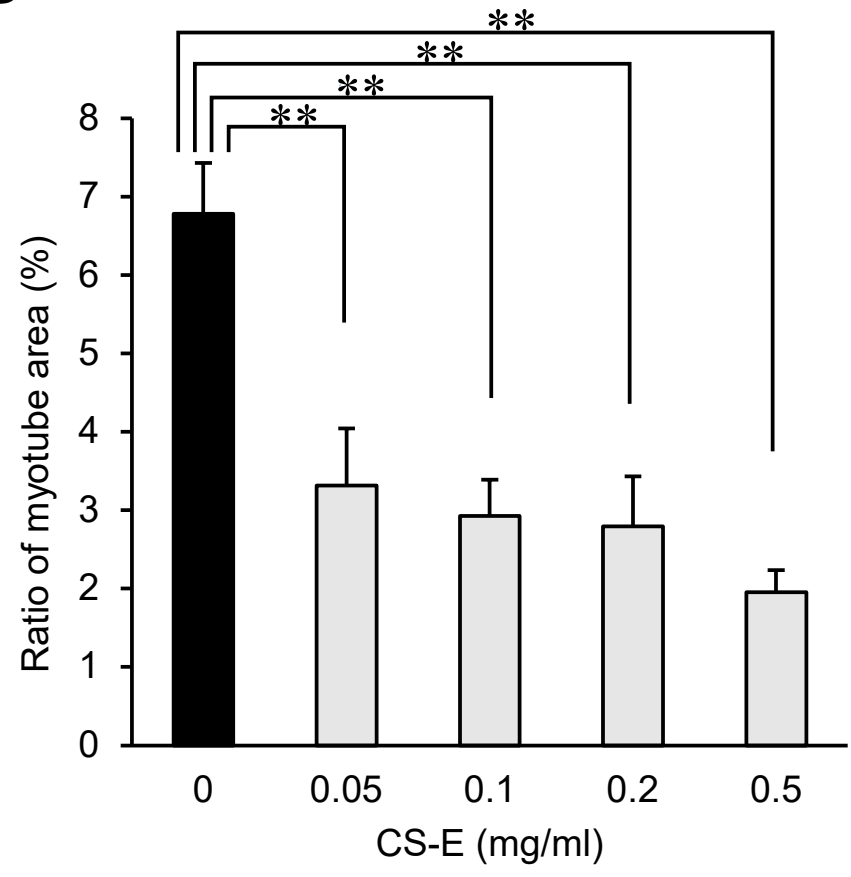

C

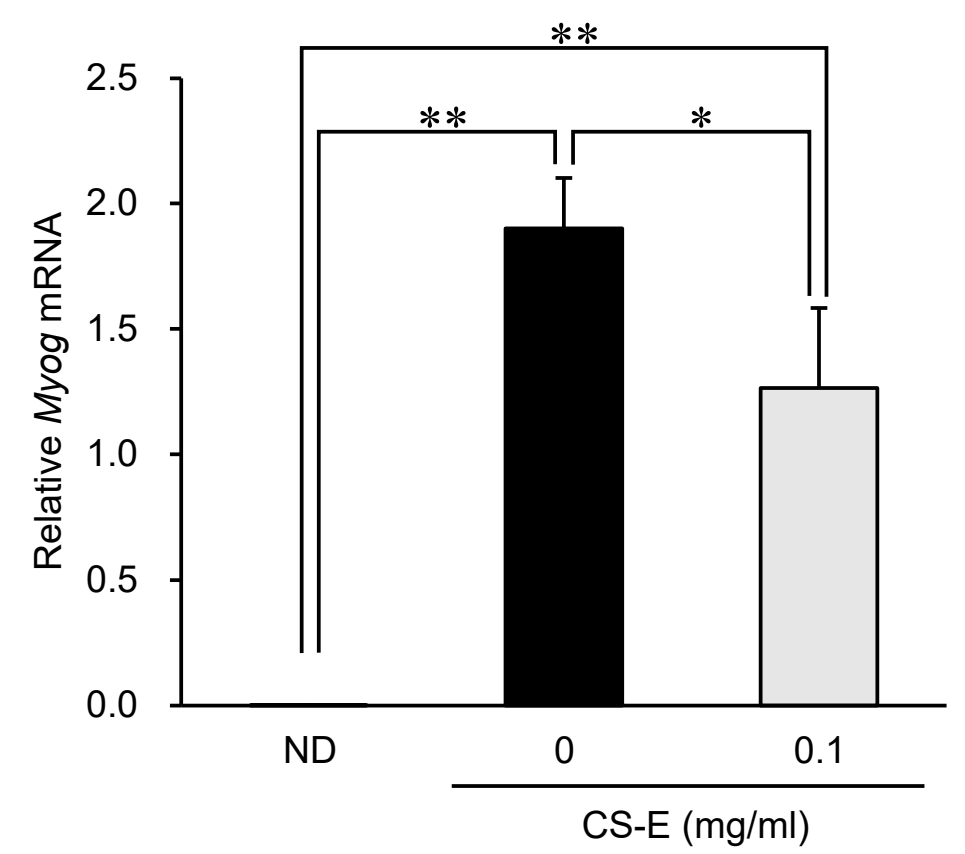


Figure 2

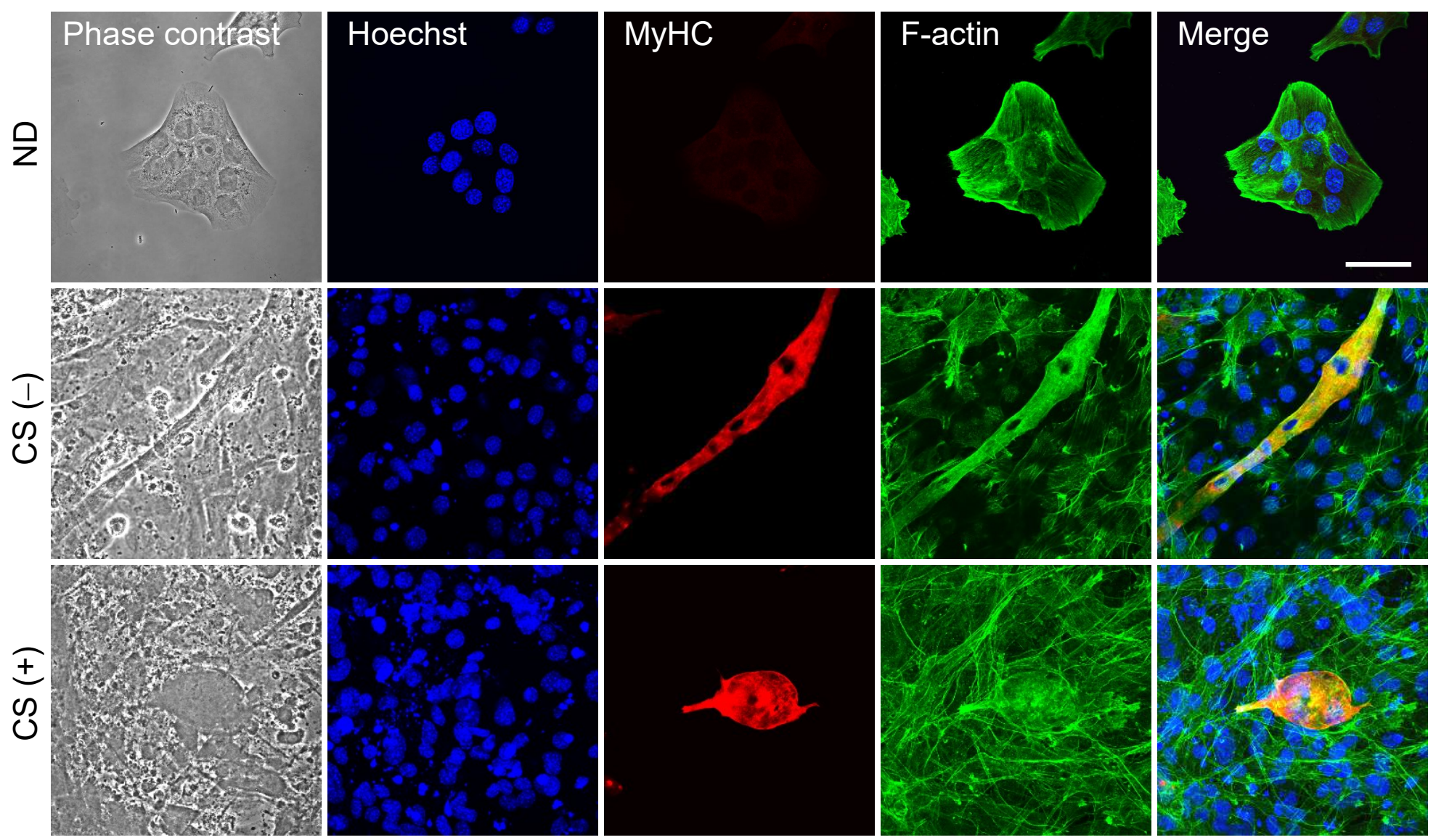


Figure 3
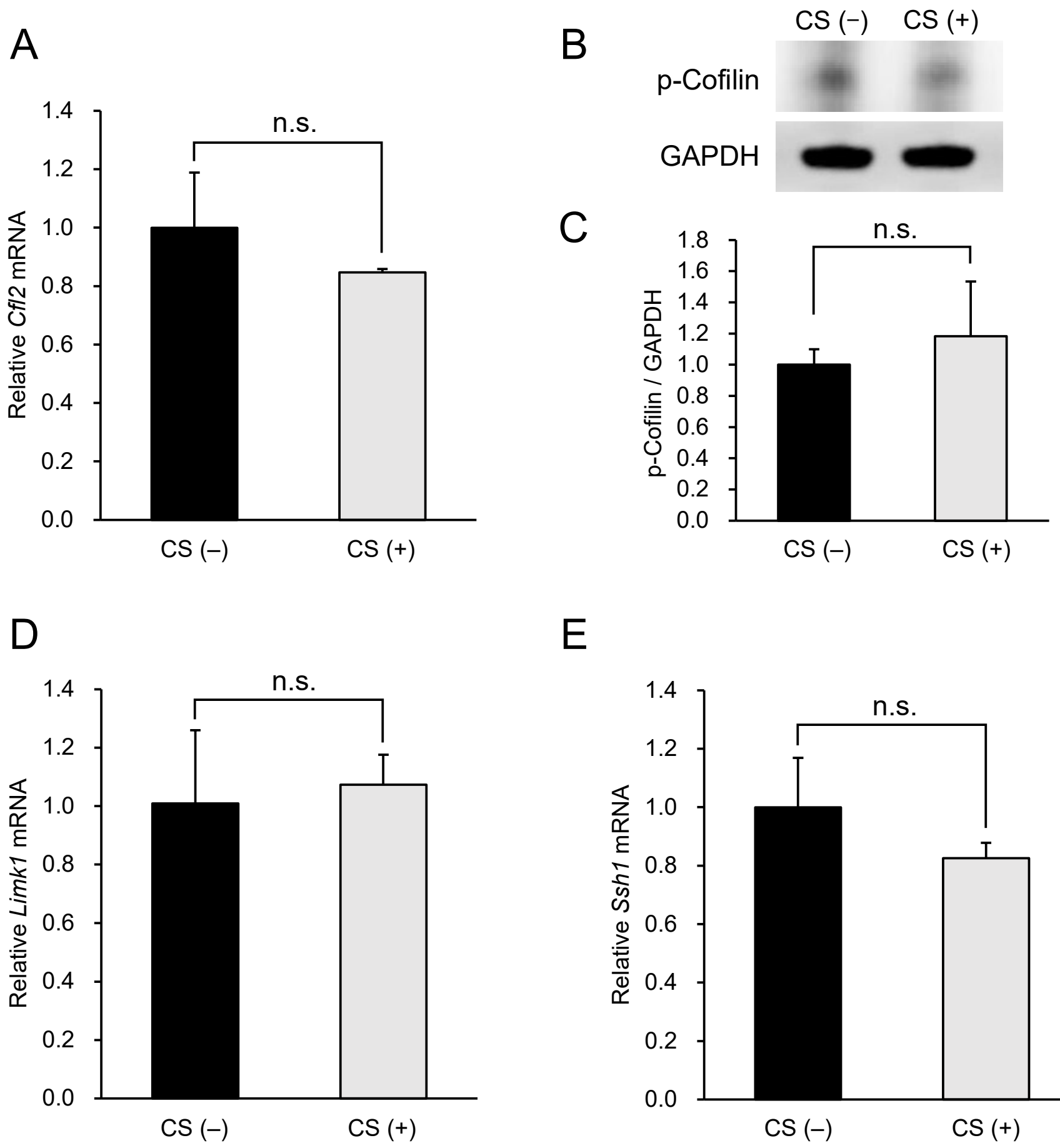

E

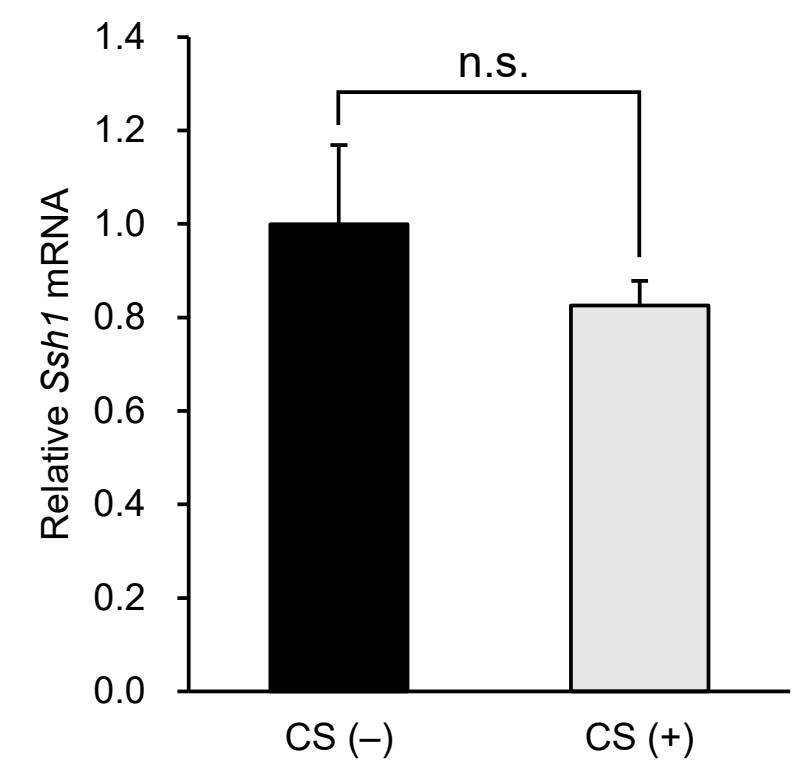


Figure 4

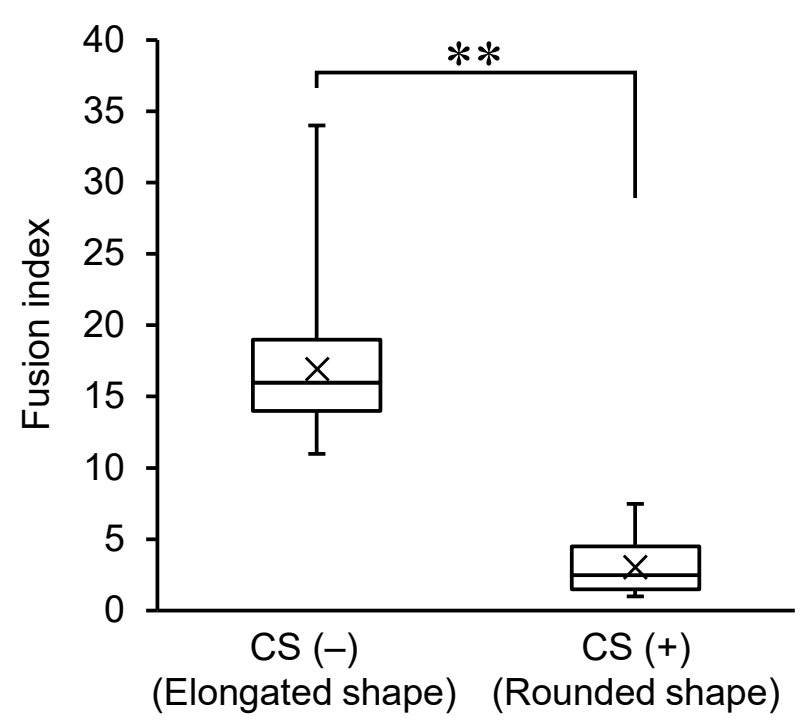


Figure 5

A

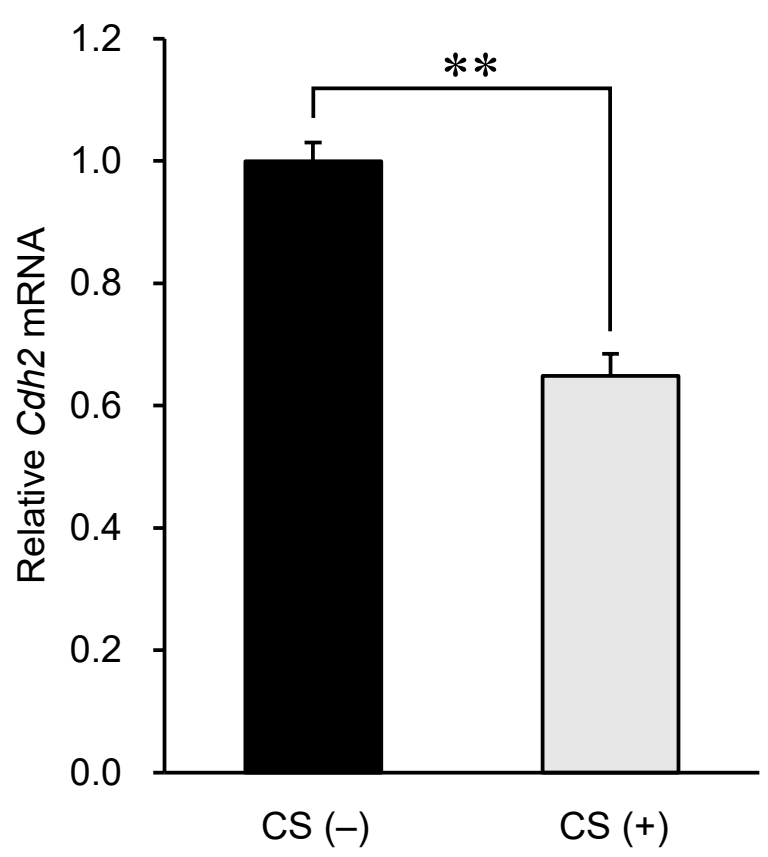

B

$\mathrm{N}$-cadherin

C

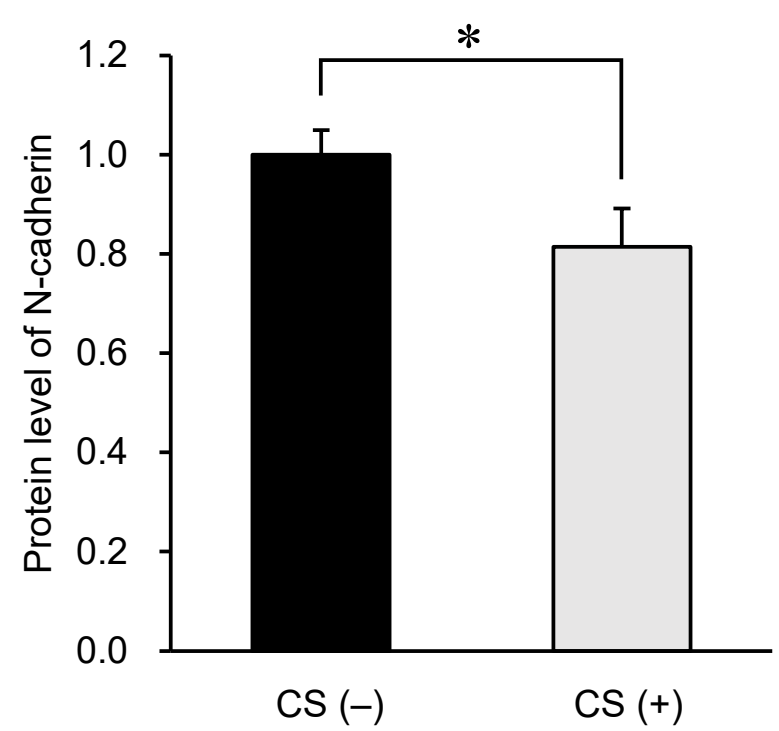


Figure 6
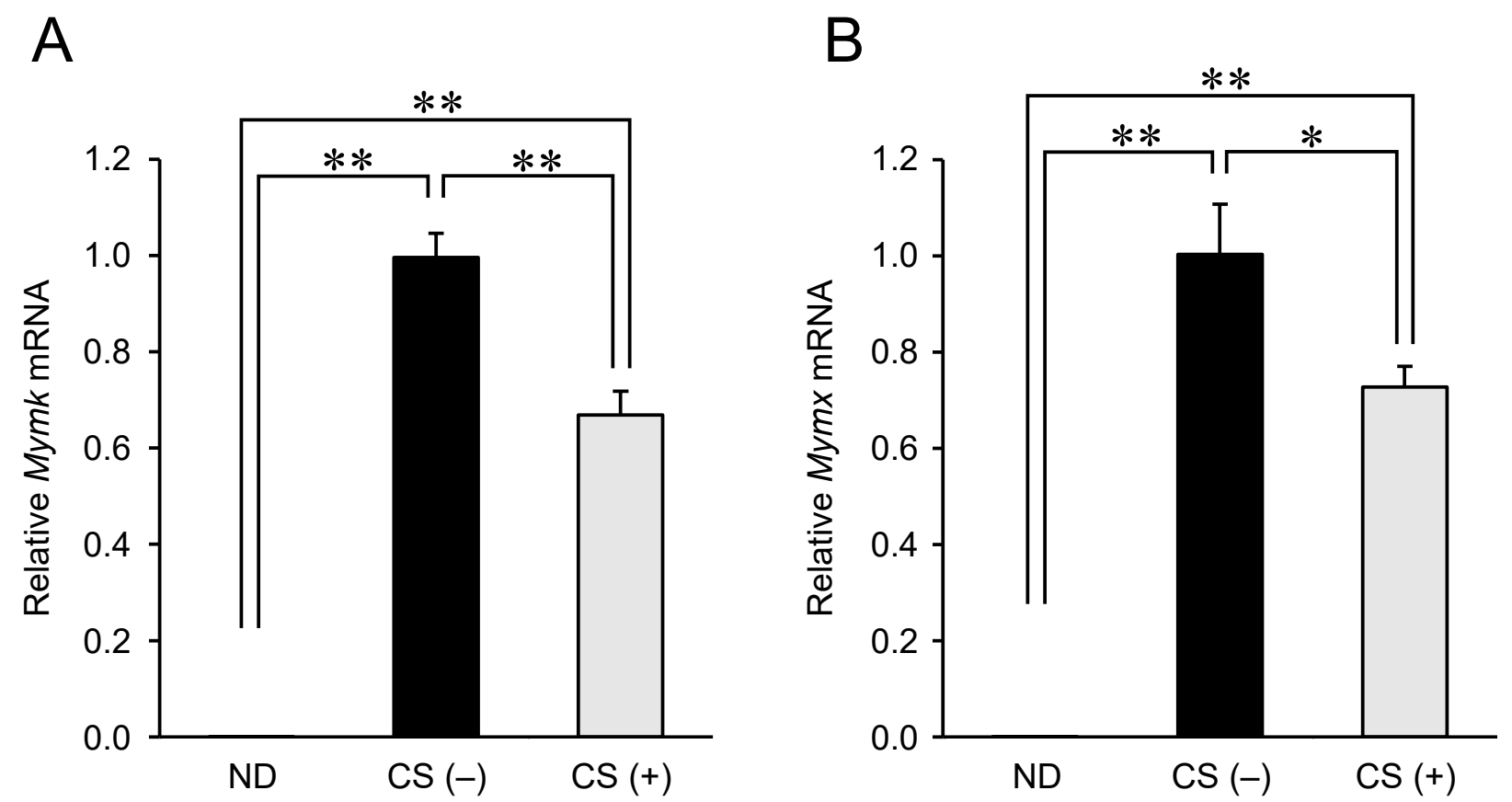
Figure 7

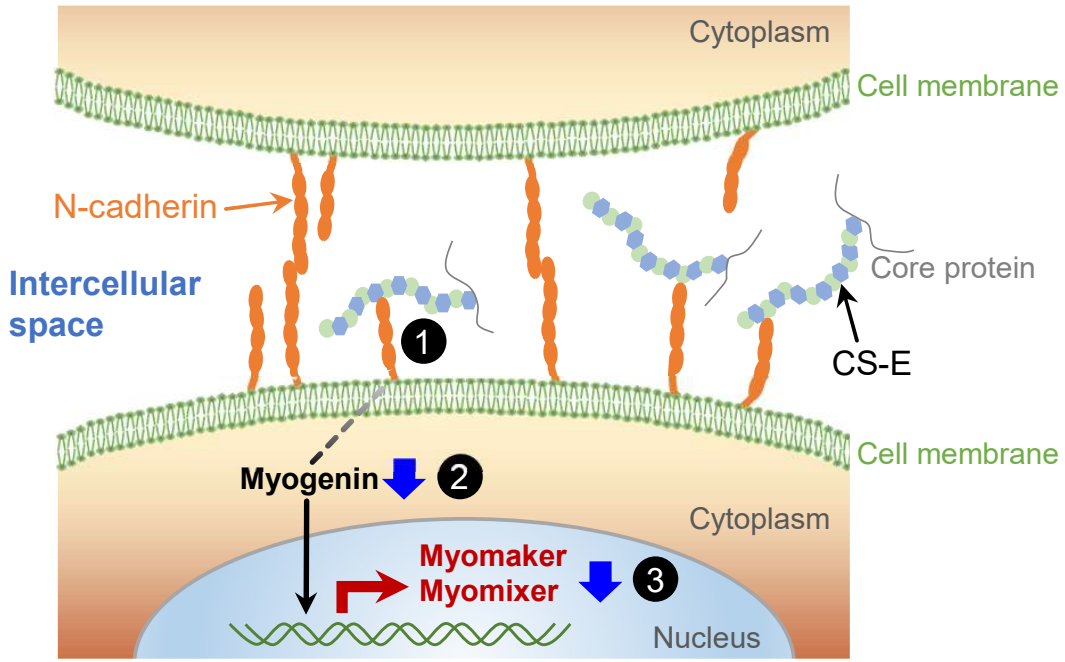


Table 1. Primer sequences for quantitative RT-PCR

\begin{tabular}{|c|c|c|c|}
\hline Gene & Primer Sequence & $\begin{array}{c}\text { Amplicon size } \\
\text { (bp) }\end{array}$ & Cross Intron \\
\hline \multirow[t]{2}{*}{ Myog } & 5'- CCTTGCTCAGCTCCCTCA -3' & 94 & no \\
\hline & 5'- TGGGAGTTGCATTCACTGG -3' & & \\
\hline \multirow[t]{2}{*}{$C f l 2$} & 5'- TGTTGCCTCTGAATGATTGC -3' & 218 & no \\
\hline & 5'- GCGGTCCTTAATATCGTCCA -3' & & \\
\hline \multirow[t]{2}{*}{ Limk1 } & 5'- TGCTCAAGTTCATCGGAGTG -3' & 178 & no \\
\hline & 5'- TTCATCGAATGGAGGTAGGC -3' & & \\
\hline \multirow[t]{2}{*}{$\operatorname{Ssh} 1$} & 5'- CCTGCGTTGTGAAGACAGAA -3' & 213 & no \\
\hline & 5'- CCATCGAGGTGAATCTTGGT -3' & & \\
\hline \multirow[t]{2}{*}{ Mymk } & 5'- ATCGCTACCAAGAGGCGTT -3' & 107 & no \\
\hline & 5'- CACAGCACAGACAAACCAGG -3' & & \\
\hline \multirow[t]{2}{*}{ Mymx } & 5'- GAGGCTCTGCTGAGCTGTCT -3' & 157 & no \\
\hline & 5'- AGTACTTTGATGGGCGTTGC -3' & & \\
\hline \multirow[t]{2}{*}{$C d h 2$} & 5'- CTGGGACGTATGTGATGACG -3' & 150 & yes \\
\hline & 5'- TGATGATGTCCCCAGTCTCA -3' & & \\
\hline \multirow[t]{2}{*}{ Gapdh } & 5'- AGGTCGGTGTGAACGGATTTG -3' & 123 & yes \\
\hline & 5'- TGTAGACCATGTAGTTGAGGTCA -3' & & \\
\hline
\end{tabular}

